results seem to indicate that educational programmes have to be repeated about every second year, if long term effects are to be expected.

Bebbington reported that the economic impact of new medical treatments are likely to become increasingly important in the future. He and professor B. Jönsson had adopted two approaches. In the first, they used a top-down cost-of-illness methodology to arrive at a conservative estimate for the direct costs to the nation of depressive illness, giving an overview of its economic impact. In the second approach, a simulation model compared the cost of old and new antidepressant therapies. The older tricyclic antidepressants were represented by imipramine, while the comparison treatment was with paroxetine, a serotonin reuptake inhibitor. The results of the costeffectiveness model suggest that an expensive drug may in practice be the cheaper option, when compliance is poor with an inexpensive drug, since treatment failure is a major component of the cost of treatment. Natziandreu et al reported that recurrent depression results in significant costs to society and the individual in monetary and quality of life terms, but that these costs are often unrecognised. Recent studies have demonstrated that recurrent depression has a greater impact than other common debilitating conditions such as hypertension or arthritis. Maintenance treatment with sertraline has been shown to reduce the recurrence of depression effectively, but the economic effects of maintenance versus episodic treatment have yet to be examined. Maintenance treatment with sertraline resulted in improvement in patients' well-being and social functioning, as measured by "quality-adjusted life years' (QALYs), in comparison to episodic treatment. The cost of this improvement was approximately $\$ 4000$ (US) which is considerably less than, e.g. the chronic treatment of severe hypertension at $\$ 16,000$ (US) per QALY.

Paul Kind from the Centre for Health Economics, University of York stated that an understanding of the costs and benefits of existing forms of treatment is an essential requirement to the economic appraisal of new forms of therapy. Such comparative information is not always immediately to hand, since many factors combine to complicate the task of establishing these baseline costs, e.g. the limited availability of data in the public domain, and the underlying absence of useful measures of outcome. In response to these difficulties, a model has been developed which identifies the principal elements in management and treatment of depression.

\title{
Ninth International Psychiatric Conference of the Pakistan Psychiatric Society
}

\author{
M. FarRuKh HussaIn, Consultant Psychiatrist, St Augustine's Hospital, Canterbury, \\ Kent CT4 7LL; and M. AfZal JAVED, Consultant Psychiatrist, Mayo Hospital \\ Lahore, Pakistan
}

The Pakistan Psychiatric Society held its ninth international conference at the Pearl-Continental Hotel, Peshawar, from 8-10 December 1992. This biannual event was attended by more than 300 participants, 45 of whom were from abroad (including UK, USA, Australia, Germany, Switzerland, and Turkey).

The main theme of the conference was institutional $v$. community care. The conference debate was enlivened through a satellite linked with Professor Leonard Stein in Madison and Dr D. McGill (New York). Institutional care was proposed by Professor M. R. Chaudry (Lahore) and seconded by Dr Peter Hall (Worcester). The proposer for community care was Professor M. H. Mubbashar (Islamabad) and the seconder Dr Ian Pullen (Edinburgh). Professor I. A. K. Tareen and Professor Andrew Sims acted as discussants. The consensus was that the debate represented a false dichotomy and that both types of care were required and needed to be adequately funded.

A second important topic was Pakistan mental health legislation. The 1912 Lunacy Act (dating from the era of the British Raj) is still in force. Replacement legislation has been discussed over the past decade. A new draft Pakistan Mental Health Act 1992, similar to current British legislation, was discussed by a panel representing psychiatrists from the UK (Professor $R$. Bluglass and Dr M. Humphreys), Australia (Dr D. Greig) and Switzerland (DrV. Dittman). There was a vigorous discussion from the floor with a conclusion that the draft should be supported with certain important amendments.

The conference presented an unusual opportunity for psychiatrists from different cultures and health care systems to exchange views. It is hoped that the knowledge of Pakistan psychiatric services gained by those from the UK will help provide more appropriate training for trainees from Pakistan who take part in the Overseas Doctors Training Scheme.

Post conference tours to the Khyber Pass and places of historical interest were well supported and much appreciated by the foreign delegates.

The next International Conference of the Pakistan Psychiatric Society will take place in Lahore in December 1994. 